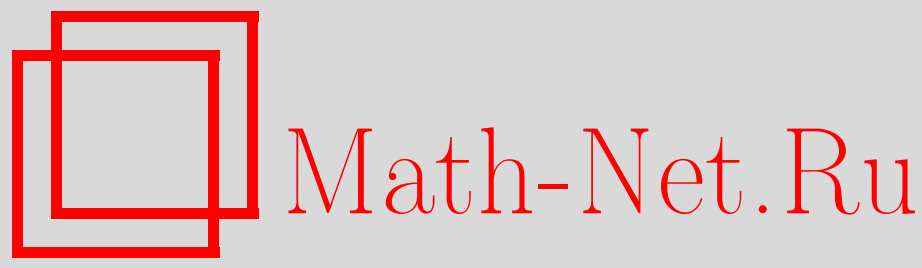

В. Н. Родионов, Г. А. Кравцова, А. М. Мандель, Волновая функция и распределение токов вероятности связанного электрона, движущегося в однородном магнитном поле, ТМФ, 2010, том 164, номер 1, 157-171

DOI: https://doi.org/10.4213/tmf6530

Использование Общероссийского математического портала Math-Net.Ru подразумевает, что вы прочитали и согласны с пользовательским соглашением http://www . mathnet.ru/rus/agreement

Параметры загрузки:

IP: 35.173 .219 .12

26 апреля 2023 г., 06:49:34

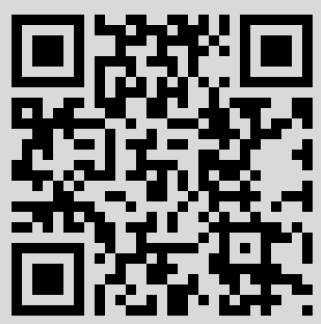




\title{
ФИЗИКА
}

Том 164, № 1

июль, 2010

\author{
2010 г. В. Н. Родионов ${ }^{*}$, Г. А. Кравцова ${ }^{\dagger}$, А. М. Мандель
}

\section{ВОЛНОВАЯ ФУНКЦИЯ И РАСПРЕДЕЛЕНИЕ ТОКОВ ВЕРОЯТНОСТИ СВЯЗАННОГО ЭЛЕКТРОНА, ДВИЖУЩЕГОСЯ В ОДНОРОДНОМ МАГНИТНОМ ПОЛЕ}

\begin{abstract}
Изучаются эффекты воздействия электромагнитных полей на нерелятивистские заряженные спинорные частицы, связанные короткодействующим потенциалом. Проведен анализ точного решения уравнения Паули для электрона, движущегося в потенциальном поле, определяемом трехмерной $\delta$-ямой, в присутствии интенсивного магнитного поля. Получены асимптотики этого решения для различных значений параметров задачи. Кроме того, рассмотрены токи вероятности для электрона и их зависимость от магнитного поля. Показано, что учет спина в рамках нерелятивистского подхода позволяет корректно учесть влияние магнитного поля на электрический ток. Полученные зависимости распределения тока, являющегося экспериментально наблюдаемой величиной, могут непосредственно проявляться, например, в процессах рассеяния.
\end{abstract}

Ключевые слова: связанный электрон, магнитное поле, распределение тока вероятности.

\section{1. ВВОДНЫЕ ЗАМЕЧАНИЯ.}

Подробное изучение эффектов воздействия внешних электромагнитных полей на связанные нерелятивистские заряженные частицы систематически проводится уже на протяжении нескольких десятилетий (см., например, обзоры [1], [2]). Однако, хотя эта проблема имеет весьма длительную историю, ряд вопросов по-прежнему нуждается в дополнительном исследовании. К таким вопросам, в частности, можно отнести анализ влияния интенсивного магнитного поля на связанные заряженные частицы при корректном учете их спиновых состояний.

${ }^{*}$ Российский государственный геологоразведочный университет им. С. Орджоникидзе, Москва, Россия. E-mail: vnrodionov@mtu-net.ru

${ }^{\dagger}$ Московский государственный университет им. М.В. Ломоносова, Москва, Россия. E-mail: krav@dio.ru

${ }^{\ddagger}$ Московский авиационный институт (Государственный технический университет), Москва, Россия. E-mail: arkadimandel@mail.ru 
Существует распространенное представление о том, что последовательный учет спина заряженной частицы требуется только при исследовании релятивистских эффектов. Но, как было показано в работах [3], [4], в интенсивном магнитном поле это не всегда так. Отметим, что попытки анализа спиновых эффектов в нерелятивистском приближении проводились неоднократно. Для этого использовались модели, точно учитывающие влияние поля на частицу. Подчеркнем, что богатый опыт в описании бесспиновых частиц, связанных $\delta$-образным потенциалом, в интенсивных электромагнитных полях, был накоплен именно благодаря использованию непертурбативного математического подхода. Разработанные методы, основанные на точных аналитических решениях уравнения Шредингера [1], [2], [5], применялись и к исследованию процессов с учетом спина частиц. Однако из за сложности математических оценок в случае спинорных частиц получаемые выводы не всегда представляются достаточно убедительными. По нашему мнению, наиболее корректными с точки зрения необходимости учета спина частиц представляются работы, в которых проводится непосредственное сравнение влияния поля на поведение спинорных и скалярных частиц [2], [4], [6]. Для этих целей в указанных работах наряду с решениями уравнения Шредингера используются и решения уравнения Паули. В настоящей статье мы рассмотрим эффекты, обусловленные действием интенсивного постоянного однородного магнитного поля на электрон, связанный короткодействующим потенциалом.

Хорошо известно, что потенциал в виде $\delta$-ямы является широко распространенной моделью, описывающей многоэлектронные атомы и в особенности отрицательные ионы. Под действием поля энергетические уровни электрона испытывают сдвиг, величина которого определяется из трансцендентного уравнения для энергии. Отметим, что в случае скалярной частицы эта задача анализировалась еще в работе [5]. Аналогичная задача для электрона с учетом его спиновых состояний в магнитном поле была корректно решена совсем недавно в статье [6].

Основная цель настоящей работы заключается в анализе точного решения уравнения Паули для электрона, движущегося в потенциальной яме, которая определяется трехмерной $\delta$-функцией, в присутствии интенсивного магнитного поля, а также в получении асимптотик этого решения при различных значениях параметров задачи. Кроме того, мы рассматриваем токи вероятностей для данной частицы и их зависимость от спина и магнитного поля.

\section{2. ТОЧНОЕ АНАЛИТИЧЕСКОЕ РЕШЕНИЕ УРАВНЕНИЯ ПАУЛИ ДЛЯ ПРИТЯГИВАЮЩЕЙ ТРЕХМЕРНОЙ $\delta$-ЯМЫ И ЕГО АСИМПТОТИКИ}

Точное аналитическое выражение для волновой функции заряженной скалярной частицы в связанном $\delta$-потенциалом состоянии, движущейся в интенсивном магнитном поле, было найдено в работе [5] с помощью функции Грина соответствующего уравнения Шредингера. Отметим, что функция Грина скалярной частицы во внешнем магнитном поле представлена в классической монографии [7] (см. также статью [8]). Как известно, учет спина электрона можно провести, например, путем пе- 
рехода к нерелятивистскому пределу в решениях уравнения Дирака, описывающих движение спинорной частицы в заданном внешнем поле [9]. Полученная функция Грина является решением уравнения Паули с $\delta$-источником и может быть представлена в виде интеграла по времени [10]. Этот интеграл, определяющий функцию Грина, допускает виковский поворот в нижнюю комплексную полуплоскость по переменной $t$ (см., например, книгу [11]). Данная операция делает интеграл чисто действительным. В результате стационарную функцию Грина можно представить в виде

$$
\begin{aligned}
G_{\mathrm{W}}(\vec{r}, \overrightarrow{0})= & -i\left(\frac{m}{2 \pi}\right)^{3 / 2} \frac{\omega_{H}}{2} e^{-i m \omega_{H} x y / 4} \times \\
& \times \int_{0}^{\infty} \frac{d t}{t^{1 / 2}} \operatorname{sh}^{-1}\left(\frac{\omega_{H} t}{2}\right) e^{S / \hbar}\left(\begin{array}{c}
1 / 2+s \\
1 / 2-s
\end{array}\right),
\end{aligned}
$$

где $m$ - масса частицы, $\omega_{H}=e H / m c$ - циклотронная частота, $e$ - модуль заряда частицы, $H$ - напряженность однородного магнитного поля, ориентированного вдоль оси $z, s= \pm 1 / 2$ - спиновое число, а показатель экспоненты под интегралом по существу представляет собой классическую функцию действия

$$
S=-\frac{m z^{2}}{2 t}-\frac{1}{4} m \omega_{H} \rho^{2} \operatorname{cth}\left(\frac{\omega_{H} t}{2}\right)+\left(W-s \cdot \hbar \omega_{H}\right) t
$$

(хотя формально показатель экспоненты содержит постоянную Планка, благодаря сдвигу энергии связанной спинорной частицы в магнитном поле [6] эта зависимость исчезает). В формулах (1), (2) $x, y, z$ - декартовы координаты, $\rho^{2}=x^{2}+y^{2}, W<0$ полная энергия связанной частицы. Заметим, что (1), (2) - обычный пропагатор заряженной частицы, движущейся в магнитном поле, продолженный в область отрицательных энергий.

Запишем пространственную часть волновой функции в виде

$$
\psi(\vec{r})=\tilde{N} e^{-i m \omega_{H} x y / 4} \int_{0}^{\infty} \frac{d t}{t^{1 / 2}} \operatorname{sh}^{-1}\left(\frac{\omega_{H} t}{2}\right) e^{S / \hbar},
$$

где $\tilde{N}$ - нормировочный коэффициент. Перейдем к более естественным переменным, используя характерные масштабы данной задачи. В качестве масштаба энергии примем $\left|W_{0}\right|$ - модуль энергии связи частицы в отсутствие магнитного поля. В таких единицах безразмерную энергию частицы обозначим через $w=W /\left|W_{0}\right|$. В отсутствие внешнего поля она равна -1 . Разумеется, в общем случае $w$ зависит как от внешнего поля $H$, так и от направления спина [6]. Магнитное поле удобно измерять в безразмерных единицах $h=\hbar \omega_{H} /\left|W_{0}\right|$. Естественным масштабом координат может служить длина волны де Бройля частицы при нулевом магнитном поле, $l_{0}=\hbar\left(2 m\left|W_{0}\right|\right)^{-1 / 2}$, а масштабом времени - величина $t_{0}=\hbar\left|W_{0}\right|^{-1}$.

В таких единицах для пространственной части волновой функции мы имеем

$$
\psi(\vec{r})=N e^{-i h \tilde{x} \tilde{y} / 4} \int_{0}^{\infty} \frac{d \tau}{\tau^{1 / 2}} \frac{\exp \left[-\tilde{z}^{2} /(4 \tau)-h \tilde{\rho}^{2} \operatorname{cth}(h \tau / 2) / 8-\widetilde{w} \tau\right]}{1-e^{-h \tau}},
$$


где $N$ - нормировочный коэффициент в новых переменных, $\tilde{x}, \tilde{y}, \tilde{z}, \tau$ - безразмерные координаты и время. Любопытно отметить аналогию подынтегрального выражения в (4) с классической формулой Планка для спектра абсолютно черного тела. Квантующий характер магнитного поля (как и квантовый характер излучения) не проявляется, пока показатель экспоненты $h \tau$ в знаменателе в правой части (4) достаточно мал. Обратим также внимание на фазовый множитель $e^{-i h \tilde{x} \tilde{y} / 4}$, наличие которого явно указывает на существование орбитального тока вероятности.

Для расчета нормировочного коэффициента в (4) необходимо вычислить несколько интегралов. Интегралы по координатам являются чисто гауссовыми, а интегралы по времени простой заменой сводятся к обобщенной дзета-функции Римана $\zeta(3 / 2, \varepsilon)$. В результате имеем

$$
N=\frac{h^{5 / 4}}{2 \pi l_{0}^{3 / 2}} \zeta^{-1 / 2}\left(\frac{3}{2}, \frac{\widetilde{w}}{h}\right),
$$

где $\widetilde{w}=w_{\mathrm{sh}}-w$ - модуль так называемой эфбективной энергии частицы. Появление параметра $\widetilde{w}$ обусловлено тем, что нижняя граница сплошного спектра в магнитном поле испытывает сдвиг наряду со сдвигом самих связанных состояний $w$. Вследствие этого эффективные энергии связи отсчитываются уже от новых границ, определяемых выражением $w_{\mathrm{sh}}=(s+1 / 2) h[6]$. Из этого соотношения также непосредственно следует, что сдвиг сплошного спектра $w_{\mathrm{sh}}$ явно зависит как от величины магнитного поля, так и от ориентации спина частицы. Однако, как показано в работе [6] на примере слабых $(h \ll 1)$ и сильных $(h \gg 1)$ полей, величина $\widetilde{w}$ от спина не зависит.

Легко видеть, что интеграл в (4) относится к интегралам типа Лапласа [12], [13]. Существенная область интегрирования определяется окрестностью единственной точки максимума экспоненциальной функции. Рассмотрим вклад седловой точки в интеграл (4), записанный в виде

$$
\int_{0}^{\infty} \frac{d u}{u^{1 / 2}} \frac{e^{-h g(u)}}{1-e^{-u}}, \quad g(u)=\frac{\tilde{z}^{2}}{4 u}+\frac{\tilde{\rho}^{2}}{8} \operatorname{cth}\left(\frac{u}{2}\right)+\frac{\widetilde{w}}{h^{2}} u .
$$

Здесь $u=h \tau$, а седловая точка является корнем уравнения

$$
B u^{2}-A=\frac{u^{2}}{\operatorname{sh}^{2}(u / 2)}, \quad A=4 \tilde{z}^{2} \tilde{\rho}^{-2}, \quad B=16 \tilde{w} \tilde{\rho}^{-2} h^{-2} .
$$

Графическая иллюстрация поиска решения этого трансцендентного уравнения приведена на рис. 1, где изображены семейство парабол, отвечающих левой части уравнения (7) при разных значениях параметра $h$ (сплошные линии) и график функции в правой части уравнения (штрихпунктирная кривая). Нетрудно убедиться, что пересечение ветви параболы с монотонной функцией $u^{2} \mathrm{sh}^{-2}(u / 2)$ может произойти в различных областях значений параметра интегрирования $u$. Так, в области $u \ll 1$ правая часть уравнения мало отличается от константы, и для седловой точки, являющейся в этом случае корнем квадратного уравнения, получаем

$$
u_{0}=\frac{h \sqrt{\tilde{\rho}^{2}+\tilde{z}^{2}}}{2 \sqrt{\widetilde{w}}} .
$$




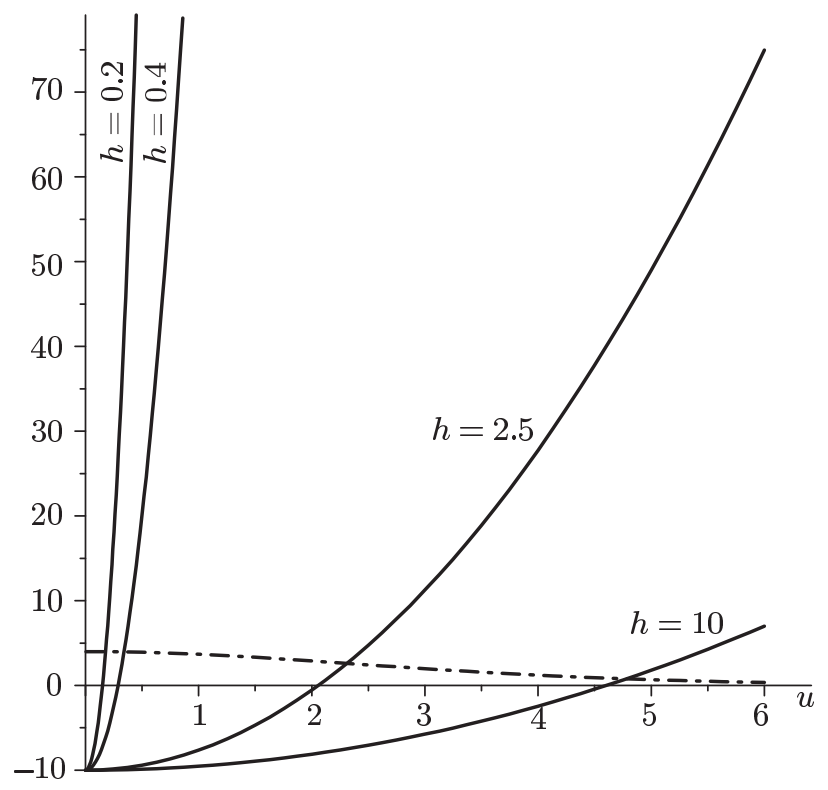

Рис. 1. Решение трансцендентного уравнения (7) в плоскости $z=1.6$ при $\rho=1$. Сплошные кривые - левая часть уравнения (7) для различных значений магнитного поля, штрихпунктирная кривая - график правой части уравнения (7).

В другом предельном случае $u \gg 1$ правая часть уравнения (7) практически обращается в нуль, и для точки перевала мы имеем

$$
u_{0}=\frac{h \tilde{z}}{2 \sqrt{\widetilde{w}}}
$$

Наконец, в промежуточной области решение уравнения (7) можно записать в приближенном виде

$$
u_{0} \approx\left[\frac{\tilde{z}^{2} h^{2}}{8 \widetilde{w}}-6+6\left[1+\frac{\tilde{z}^{2} h^{2}}{24 \widetilde{w}}+\frac{\tilde{\rho}^{2} h^{2}}{12 \widetilde{w}}+\frac{\tilde{z}^{4} h^{4}}{2304 \widetilde{w}^{2}}\right]^{1 / 2}\right]^{1 / 2}
$$

Легко видеть, что данное решение в пределах слабых $(h \ll 1)$ и сильных $(h \gg 1)$ полей переходит в (8) и (9) соответственно. Оценка интеграла (6), полученная методом перевала, при этом записывается как

$$
I(\tilde{z}, \tilde{\rho}) \approx 2 \sqrt{\frac{\pi}{h}}\left[\frac{4 \tilde{z}^{2}}{u_{0}^{2}} \operatorname{sh}^{2}\left(\frac{u_{0}}{2}\right)+\frac{\tilde{\rho}^{2} u_{0}}{2} \operatorname{cth}\left(\frac{u_{0}}{2}\right)\right]^{-1 / 2} e^{\left(u_{0} / 2\right)-h g\left(u_{0}\right)} .
$$

Перейдем теперь к изучению волновой функции в пределах слабых и сильных полей. Из дальнейших рассуждений будет видно, что сами эти понятия в рассматриваемой задаче нуждаются в некотором уточнении. Если $h \ll 1$, а $\tilde{\rho}$ и $\tilde{z}$ не слишком 


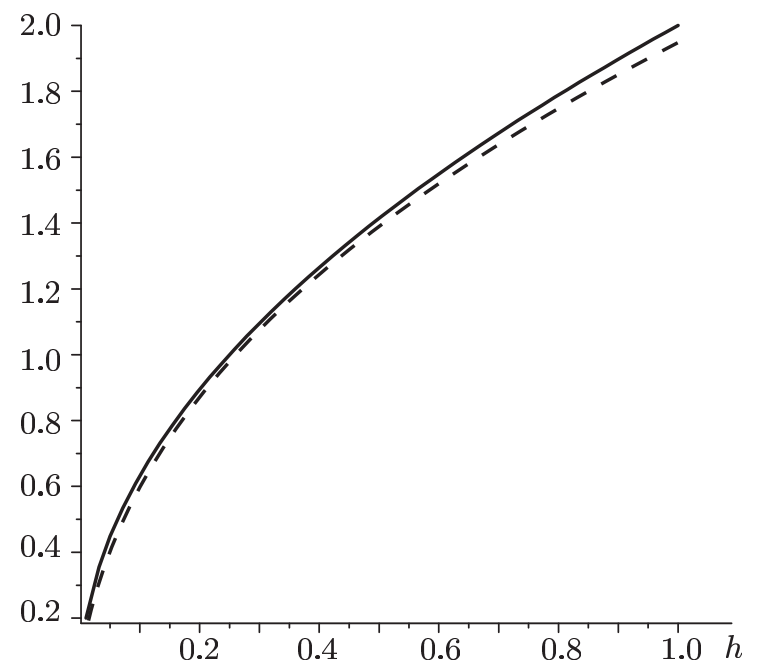

Рис. 2. Точная зависимость от магнитного поля $h$ функции $\zeta(3 / 2, \widetilde{w} / h)$ (штриховая линия) и зависимость, определяемая правой частью формулы (13) (сплошная линия).

велики, то решение (8) приводится к виду

$$
u_{0}=\frac{h \tilde{r}}{2 \sqrt{\widetilde{w}}} \ll 1,
$$

где $\tilde{r}=\sqrt{\tilde{z}^{2}+\tilde{\rho}^{2}}-$ безразмерная радиальная координата. Фактически в рассматриваемом пределе это выражение определяет сферический тип симметрии электронного облака.

Как уже говорилось во введении, в работе [6] изучено поведение связанного уровня энергии спинорной частицы, находящейся в $\delta$-яме, в магнитном поле. Важно отметить, что полученное решение имеет устойчивые асимптотики по спиновой переменной в пределах слабого и сильного полей, т.е. и в том, и в другом случае эффективная энергетическая характеристика $\widetilde{w}$ не зависит от спина. Хотя используемый в работе [6] способ расчета несколько отличается от методов, принятых в ранних работах [5], [8] для скалярных частиц, имеется полная согласованность полученных в статье [6] результатов, как в пределе слабых, так и в пределе сильных полей. В частности, согласно работе [6] при небольших значениях магнитного поля имеем $\widetilde{w} \approx 1+h / 2$, откуда непосредственно видно отсутствие зависимости $\widetilde{w}$ от спина частиц.

Учитывая изложенное выше, используем оценку для дзета-функции

$$
\zeta\left(\frac{3}{2}, \frac{\widetilde{w}}{h}\right) \sim 2 h^{1 / 2},
$$

справедливую в области $h \leqslant 1$. О применимости такой асимптотики, в частности, свидетельствуют графики, приведенные на рис. 2. Таким образом, в рассматривае- 


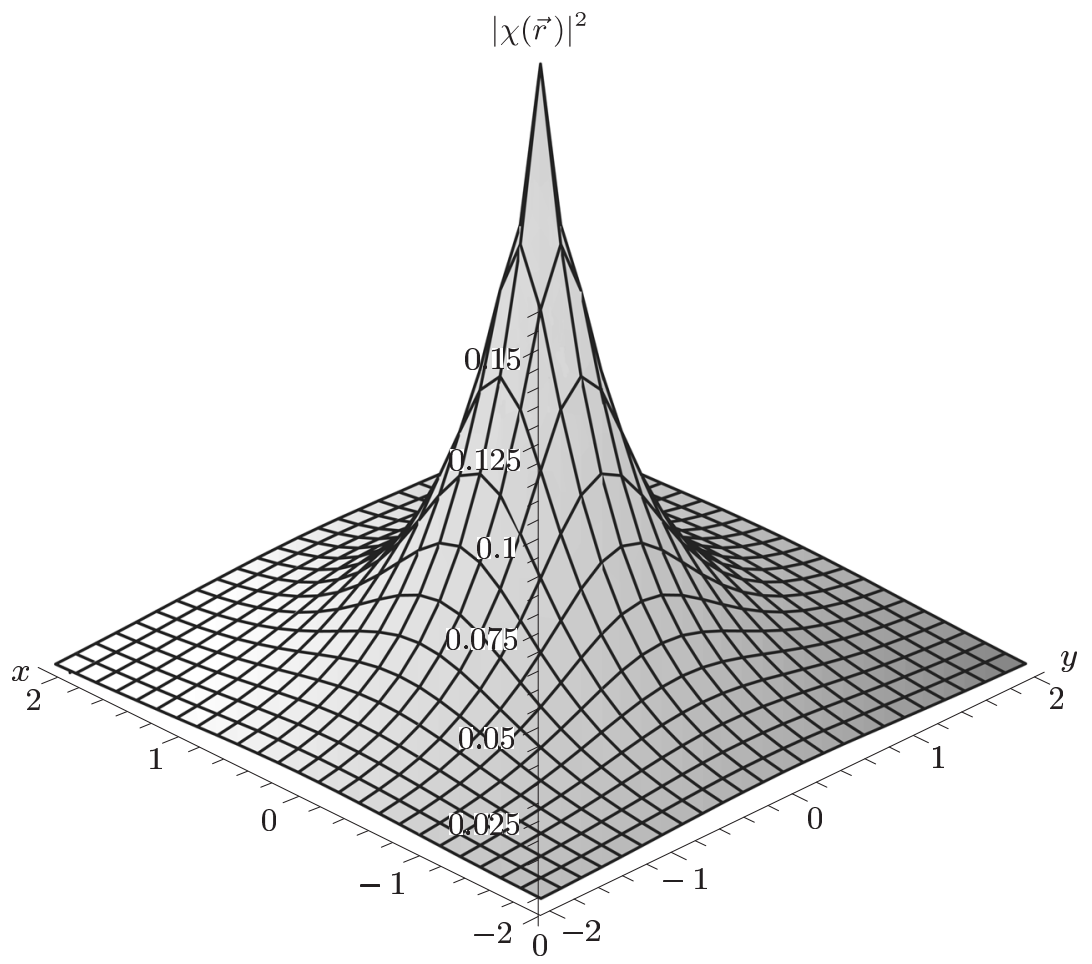

Рис. 3. Зависимость безразмерного квадрата модуля функции $\chi(\vec{r})$ от поперечной $(x)$ и продольной $(z)$ координат для полей $h \leqslant 1$.

мой области $h<1$ (и при не слишком большом удалении от $\delta$-ямы) для пространственной части волновой функции получаем выражение

$$
\psi(\vec{r}) \approx N \frac{2 \sqrt{\pi}}{h \tilde{r}} \exp \left(-\frac{i}{4} h \tilde{x} \tilde{y}-\tilde{r}\right)
$$

Для графического представления результатов удобно использовать функцию $(\vec{r})=r \psi(\vec{r})$, не имеющую особенности в нуле и определяющую пространственное распределение плотности вероятности электронного облака в сферической системе координат:

$$
d W \sim 2 \pi|\chi(\vec{r})|^{2} \sin (\theta) d \theta d r .
$$

На рис. 3 представлен график функции $|\chi(\vec{r})|^{2}$. При этом длина волны де Бройля частицы принята за единицу. Подчеркнем, что данная оценка для волновой функции получена вблизи $\delta$-ямы в пределе слабого поля. Отметим, однако, что поле порядка нескольких десятых долей от внутриатомного не является слабым в обычном представлении. 
Вдали от $\delta$-ямы даже в приближении слабого поля ситуация кардинально меняется. При $h \tilde{z} \gg 1$ из формулы (10) для точки перевала имеем

$$
u_{0}=\frac{h \tilde{z}}{2 \sqrt{\widetilde{w}}} \gg 1
$$

В результате асимптотика пространственной части волновой функции принимает вид

$$
\psi(\vec{r}) \approx N \sqrt{\frac{\pi}{1+h / 2}} \exp \left(-\frac{i}{4} h \tilde{x} \tilde{y}-\frac{h \tilde{\rho}^{2}}{8}-\tilde{z} \sqrt{1+\frac{h}{2}}\right) .
$$

Это выражение важно для качественного анализа. В данной области решение имеет осевую симметрию. Ясно, что величина поля определяет зону, где характерная для связанного s-состояния сферическая симметрия переходит в осевую симметрию, присущую волновым функциям частиц в чисто магнитном поле. Кроме того, видно, что на больших расстояниях от $\delta$-ямы даже в слабом поле основной вклад вносит только основной уровень эффективной энергии, т.е. уровень, наиболее близко расположенный к границе сплошного спектра $(1+h / 2$ в принятых нами единицах). Это полностью согласуется с выводами работ [5], [6]. Обычно, когда рассматриваются процессы со свободными частицами во внешнем магнитном поле, предполагается, что слабое поле вообще не проявляет свой квантующий характер, давая лишь малые поправки в сечения соответствующих процессов. Напротив, традиционно считается, что в сильном поле вклад вносят лишь несколько минимальных по энергии уровней [11].

В противоположность к этому в связанном состоянии любое сколь угодно слабое поле ведет себя как сильное на большом расстоянии от центра. В частности, из формулы (16) следует, что магнитное поле сжимает электронное облако не только в поперечном к полю направлении (что вполне ожидаемо), но и вдоль поля. Однако, если пренебречь сдвигом основного уровня эффективной энергии в магнитном поле, т.е. считать, что $\widetilde{w}=1$, то подобные эффекты не могут быть описаны. Отметим, что при этих упрощающих предположениях решение (16) согласуется с основной функцией, используемой в работе [14].

В сильном поле ситуация оказывается более сложной. Причина очевидна, т.к. в данном случае переходная зона от сферической к осевой симметрии лежит в области, где волновая функция существенно отлична от нуля. Используя результаты работы [6] по определению эффективной энергии электрона в сильных полях $(h \gg 1)$, $\widetilde{w} \approx 0.295 h$, рассмотрим малую окрестность $\delta$-центра. При $\tilde{r} \ll 1 / \sqrt{h}$ положение точки перевала определяется выражением (12). Для волновой функции с помощью соотношений (4), (11) имеем аналог выражения (14), но с другим значением эффективной энергии:

$$
\psi(\vec{r}) \approx N \frac{2 \sqrt{\pi}}{h \tilde{r}} \exp \left(-\frac{i}{4} h \tilde{x} \tilde{y}-\tilde{r} \sqrt{0.295 h}\right) .
$$

Таким образом, дополняя наши выводы о поведения функции на больших расстояниях в слабом поле, укажем, что любое сколь угодно сильное поле в непосредственной близости от притягивающего $\delta$-центра проявляет себя как слабое. Истинная 


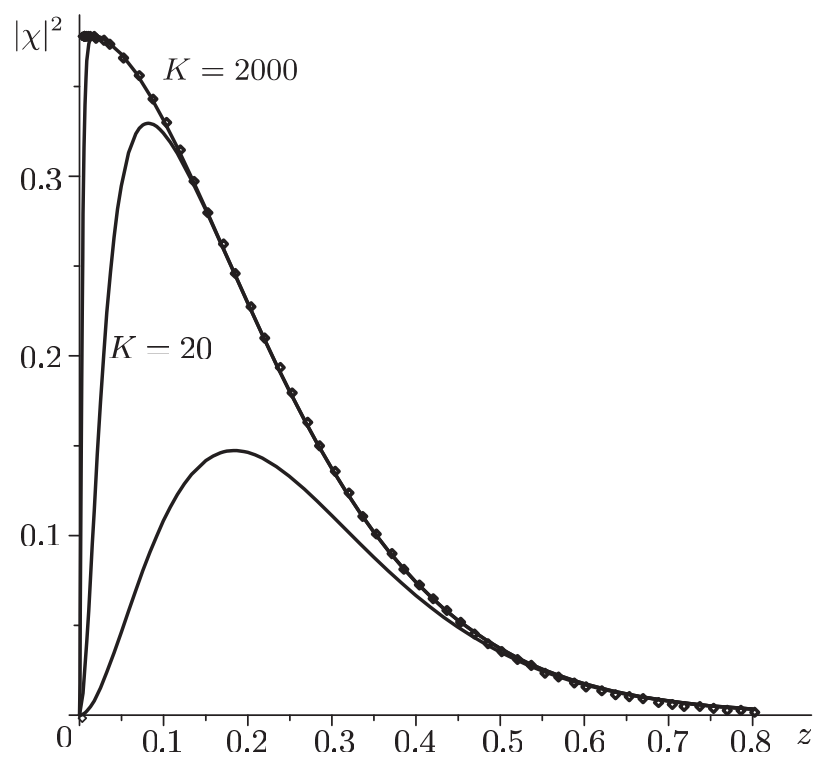

Рис. 4. Зависимость $|\chi|^{2}$ от $z$ на оси $\rho=0$ для поля $h=100$. Точки соответствуют расчету по точной формуле (4), сплошные линии (последовательно снизу вверх) определяют приближения сильного поля: вклад основного уровня (18), вклады для $K=20$ и $K=2000$, рассчитанные по формуле (20).

причина этого состоит в том, что глубина $\delta$-ямы много больше любого конечного сдвига уровня энергии в магнитном поле. Именно из того, что характер этой асимптотики не зависит от внешнего магнитного поля, и следует уравнение для энергии связанного уровня $w(h)$ [5], [6], [8].

Волновая функция становится осесимметричной в сильном поле на расстояниях $\tilde{z} \gg 1 / \sqrt{h}$. Для $h \gg 1$ при фиксированных $\tilde{\rho} \neq 0$ и $\tilde{z} \neq 0$ получаем формулу (15). Для волновой функции из соотношений (4), (11) имеем аналог формулы (16) для сильных полей:

$$
\psi(\vec{r}) \approx N \sqrt{\frac{\pi}{0.295 h}} \exp \left(-\frac{i}{4} h \tilde{x} \tilde{y}-\frac{h \tilde{\rho}^{2}}{8}-\tilde{z} \sqrt{0.295 h}\right) .
$$

Легко видеть, что здесь также вклад вносит только основной уровень эффективной энергии.

Сферически-симметричная (12) и осесимметричная (15) оценки точки максимума согласуются в окрестности прямой $\tilde{\rho}=0$. Интересно, что изначально зависимость энергии связанного уровня от магнитного поля получена авторами работы [5] с помощью предельного перехода в выражении для волновой функции именно вдоль этой линии. Поэтому для расширения области применимости полученного соотношения (18) в сторону малых $z$ можно в исходной формуле (4) разложить знаменатель как геометрическую прогрессию по парциальным эффективным уровням

$$
\widetilde{w}_{k}=\widetilde{w}+k h, \quad k=0,1,2, \ldots,
$$




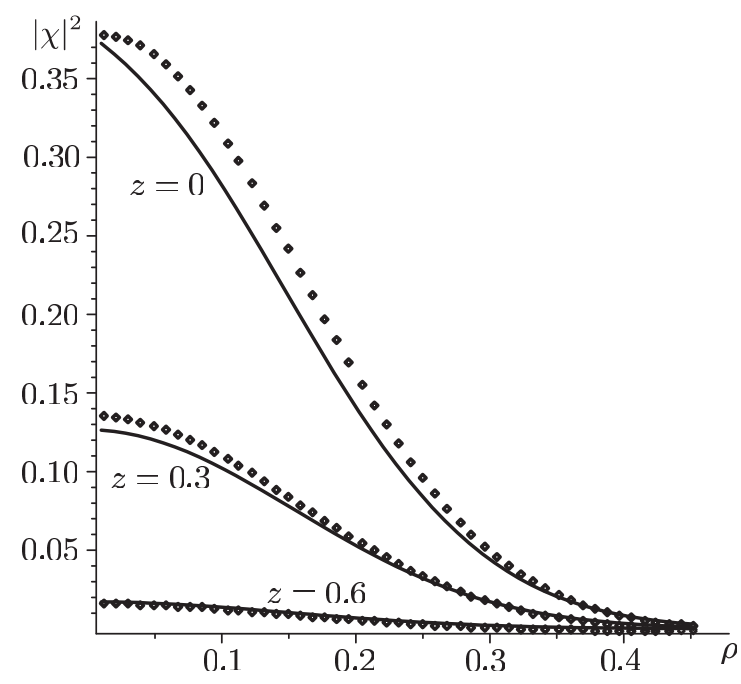

Рис. 5. Зависимость $|\chi|^{2}$ от $\rho$ для поля $h=100$ при различных $z$. Точки расчет по точной формуле (4), сплошные линии - приближения сильного поля по формулам (10), (11).

заменяющим собой уровни Ландау в чисто магнитном поле [15], и использовать для каждого слагаемого оценку (18). Волновая функция в этом случае принимает вид

$$
\psi(\vec{r}) \approx N \sqrt{\pi} \exp \left(-\frac{i}{4} h \tilde{x} \tilde{y}-\frac{h \tilde{\rho}^{2}}{8}\right) \sum_{k=0}^{K} \frac{e^{-\tilde{z} \sqrt{(0.295+k) h}}}{\sqrt{(0.295+k) h}}
$$

Легко убедиться, что, увеличивая в сумме максимальное число $K$ учтенных уровней, можно продлить область применимости полученной формулы вплоть до сколь угодно малых значений координаты $z$.

На рис. 4 квадрат модуля функции $\chi(\vec{r})$, рассчитанной с помощью точной формулы (4), сопоставлен с асимптотиками (18), (20) для поля $h=100$ на оси $\rho=0$. Ряд в решении (20) сходится тем лучше (и, следовательно, первый член ряда (18) тем ближе к точному решению), чем больше параметр $\sqrt{h} z$. Это оказывается важным, т.к. в этой области ток вероятности максимален.

На рис. 5 сопоставлены выражения для $|\chi(\vec{r})|^{2}$ при трех различных значениях $z=0,0.3,0.6$ в сильном поле $h=100$, рассчитанное по точной формуле (4) и по приближенной формуле (11) с учетом (10).

Подведем краткие итоги раздела. В слабом магнитном поле во всей значимой области изменения координат решение уравнения Паули сферически-симметрично. Оно, по сути, определяется только характером особенности в $\delta$-яме, в формирование этой особенности вносят вклад все эффективные парциальные уровни $\widetilde{w}_{k}$ (см. формулу (19)). Квантующий характер магнитного поля (т.е. ощутимость вклада отдельных уровней энергии) сказывается только далеко от центра, где волновая 
функция экспоненциально подавлена. В сильном поле характер симметрии решения меняется уже в значимой области изменения координат. Относительный вклад отдельных уровней при фиксированной интенсивности поля определяется прежде всего продольной (в направлении магнитного поля) координатой, хотя сжимается электронное облако магнитным полем прежде всего в поперечном направлении.

\section{3. ТОКИ ВЕРОЯТНОСТИ ЧАСТИЦЫ, СВЯЗАННОЙ $\delta$-ПОТЕНЦИАЛОМ, В МАГНИТНОМ ПОЛЕ}

Общее выражение для нерелятивистского тока вероятности спинора приведено в классической монографии [15]. Воспользовавшись обычными тензорными обозначениями, представим этот ток $J_{k}$ в следующей форме:

$$
J_{k}=-i \frac{\hbar}{2 m}\left[\left(\nabla_{k} \psi_{\alpha}^{*}\right) \psi_{\alpha}-\psi_{\alpha}^{*} \nabla_{k} \psi_{\alpha}\right]+\frac{e}{m c} A_{k} \psi_{\alpha}^{*} \psi_{\alpha}-\frac{\mu c}{e|s|} \varepsilon_{k p q} \nabla_{p}\left(\psi_{\alpha}^{*} \widehat{\sigma}_{\alpha \beta}^{q} \psi_{\beta}\right)
$$

где $k=1,2,3$ - пространственные координатные индексы, $\alpha=1,2$ - спиновые индексы, $\nabla_{k}$ - обычный оператор градиента, $A_{k}$ - векторный потенциал внешнего магнитного поля, $\mu$ - магнитный момент электрона, $\varepsilon_{k p q}$ - единичный абсолютно антисимметричный тензор, $\widehat{\sigma}_{\alpha \beta}^{q}$ - двоичные сигма-матрицы. Полное нормированное решение уравнения Паули можно представить в виде

$$
\psi_{\alpha}(\vec{r})=\psi(\vec{r})\left(\begin{array}{c}
1 / 2+s \\
1 / 2-s
\end{array}\right)
$$

где пространственная часть $\psi(\vec{r})$ определяется в $(4)$. Первое и второе слагаемые в (21) определяют градиентный (орбитальный) ток, а последнее соответствует спиновому току.

Проводя свертку по спиновым индексам с учетом явного вида $\sigma$-матриц, для пространственных компонент полного тока вероятности получаем

$$
\begin{aligned}
J_{x} & =-i \frac{\hbar}{2 m}\left(\frac{\partial \psi^{*}}{\partial x} \psi-\psi^{*} \frac{\partial \psi}{\partial x}\right)+\frac{e}{m c} A_{x} \psi^{*} \psi-\frac{\mu c}{e} \frac{s}{|s|} \frac{\partial}{\partial y}\left(\psi^{*} \psi\right), \\
J_{y} & =-i \frac{\hbar}{2 m}\left(\frac{\partial \psi^{*}}{\partial y} \psi-\psi^{*} \frac{\partial \psi}{\partial y}\right)+\frac{e}{m c} A_{y} \psi^{*} \psi+\frac{\mu c}{e} \frac{s}{|s|} \frac{\partial}{\partial x}\left(\psi^{*} \psi\right), \\
J_{z} & =-i \frac{\hbar}{2 m}\left(\frac{\partial \psi^{*}}{\partial z} \psi-\psi^{*} \frac{\partial \psi}{\partial z}\right)+\frac{e}{m c} A_{z} \psi^{*} \psi
\end{aligned}
$$

Для дальнейших расчетов необходимо фиксировать калибровку векторного потенциала. Выбирая его в виде $A_{x}=-H y, A_{y}=A_{z}=0$ и переходя к безразмерным координатам, получаем

$$
\vec{J}=\left(\frac{\left|W_{0}\right|}{2 m}\right)^{1 / 2} \frac{h}{2} \psi^{*} \psi(\vec{j} \tilde{x}-\vec{i} \tilde{y})+\frac{\left(2 m\left|W_{0}\right|\right)^{1 / 2}}{2 \hbar} \frac{\mu c}{e} \frac{s}{|s|} \psi^{*} \widetilde{\psi}(\vec{j} \tilde{x}-\vec{i} \tilde{y}),
$$


где $\vec{i}, \vec{j}$ - орты декартовой системы, а функция $\widetilde{\psi}(\vec{r})$ имеет вид

$$
\begin{aligned}
\widetilde{\psi}(\vec{r})= & N e^{-i h \tilde{x} \tilde{y} / 4} \int_{0}^{\infty} \frac{d \tau}{\tau^{1 / 2}} \frac{1+e^{-h \tau}}{\left[1-e^{-h \tau}\right]^{2}} \times \\
& \times \exp \left[-\frac{\tilde{z}^{2}}{4 \tau}-\frac{h}{8} \tilde{\rho}^{2} \operatorname{cth}\left(\frac{h \tau}{2}\right)-\widetilde{w} \tau\right] .
\end{aligned}
$$

Считая магнитный момент электрона равным магнетону Бора, получим

$$
\vec{J}=\left(\frac{\left|W_{0}\right|}{2 m}\right)^{1 / 2} \frac{h}{2} \psi^{*}(\psi+2 s \tilde{\psi})(\vec{j} \tilde{x}-\vec{i} \tilde{y}) .
$$

Используя точное выражение для пространственной части волновой функции (4), получаем формулу для тока вероятности при произвольной ориентации спина и интенсивности поля:

$$
\begin{aligned}
\vec{J}= & M h^{7 / 2} \zeta^{-1}\left(\frac{3}{2}, \frac{\widetilde{w}}{h}\right)(\vec{j} \tilde{x}-\vec{i} \tilde{y}) \times \\
& \times \int_{0}^{\infty} \frac{d \tau}{\tau^{1 / 2}} \frac{\exp \left[-\tilde{z}^{2} /(4 \tau)-h \tilde{\rho}^{2} \operatorname{cth}(h \tau / 2) / 8-\widetilde{w} \tau\right]}{1-e^{-h \tau}} \times \\
& \times \int_{0}^{\infty} \frac{d \tau}{\tau^{1 / 2}} \frac{\exp \left[-\tilde{z}^{2} /(4 \tau)-h \tilde{\rho}^{2} \operatorname{cth}(h \tau / 2) / 8-\widetilde{w} \tau\right]}{1-e^{-h \tau}}\left[1+2 s \cdot \operatorname{cth}\left(\frac{h \tau}{2}\right)\right],
\end{aligned}
$$

где $M=m\left|W_{0}\right|^{2} / 4 \pi^{2} \hbar^{3}$ - размерный коэффициент, не зависящий от магнитного поля.

Получим оценки для тока в приближении слабого поля. В этом случае имеем

$$
\widetilde{\psi}(\vec{r}) \approx N \cdot \frac{8 \sqrt{\pi}}{h^{2} \tilde{r}^{3}}(1+\tilde{r}) \exp \left(-\frac{i}{4} h \tilde{x} \tilde{y}-\tilde{r}\right) .
$$

Учитывая также (14) и (23), запишем выражение для тока вероятности в слабом магнитном поле:

$$
\vec{J}=M \zeta^{-1}\left(\frac{3}{2}, \frac{1}{h}+\frac{1}{2}\right) \frac{4 \pi h^{3 / 2}}{\tilde{r}^{2}} e^{-2 \tilde{r}}\left[1+2 s \cdot \frac{4(1+\tilde{r})}{h \tilde{r}^{2}}\right](\vec{j} \tilde{x}-\vec{i} \tilde{y}) .
$$

На рис. 6а изображена зависимость логарифма величины $|J| / M$ от поперечной координаты $\rho$ в различных плоскостях $z=$ const в поле $h=0.1$, рассчитанная с помощью точной (24) и приближенной (25) формул, для случая $s=-1 / 2$, т.е. для частицы со спином, ориентированным против поля. Для частицы со спином вдоль поля меняется только ориентация вектора $\vec{J}$, т.е. изменяется направление вращения частицы. Это происходит потому, что второе слагаемое в (25) вблизи $\delta$-ямы оказывается гораздо больше первого. Другими словами, в этом случае орбитальный ток вероятности пренебрежимо мал по сравнению со спиновым. Сравнимы- 


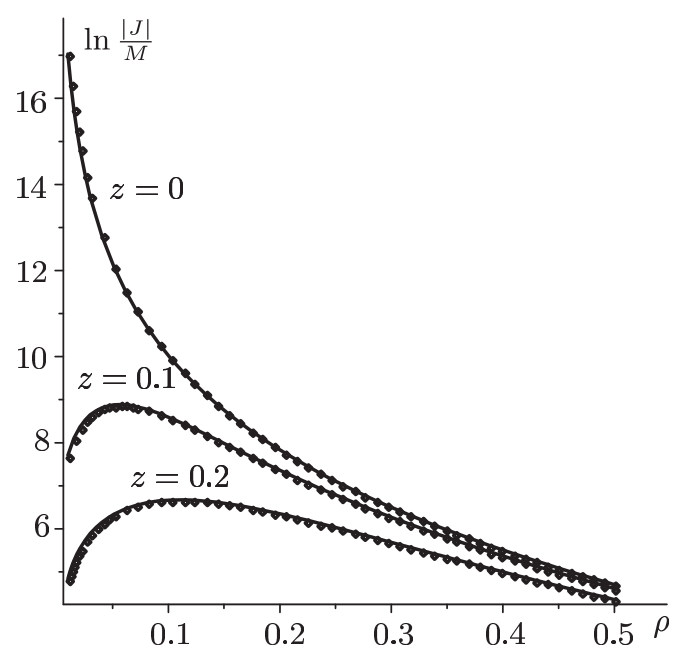

a

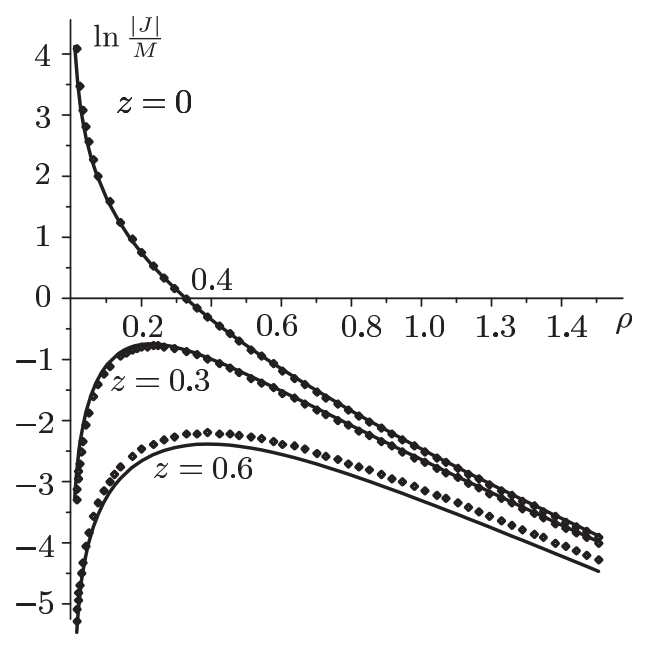

б

Рис. 6. Зависимости величины $\ln (|J| / M)$ от поперечной координаты в плоскостях $z=$ const в слабом поле $h=0.1$ для частицы со спином, направленным против поля (а), и скалярной частицы (б). Точки - расчет по точной формуле (24), сплошные линии - приближение слабого поля (25).

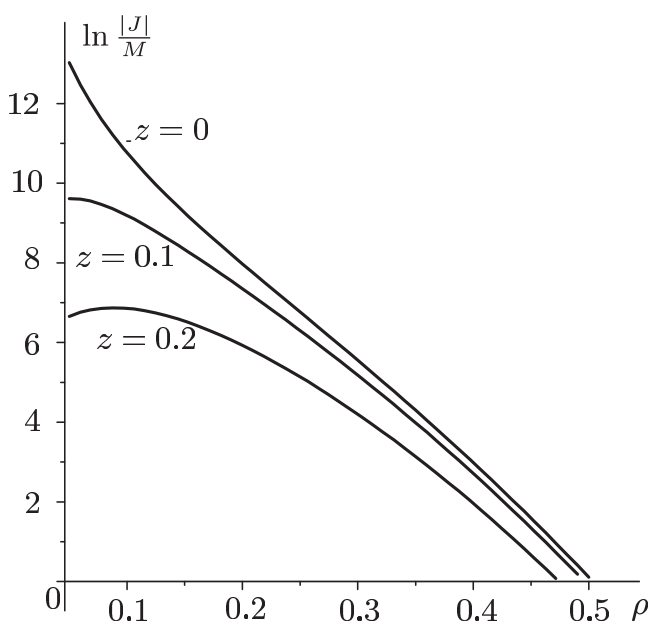

a

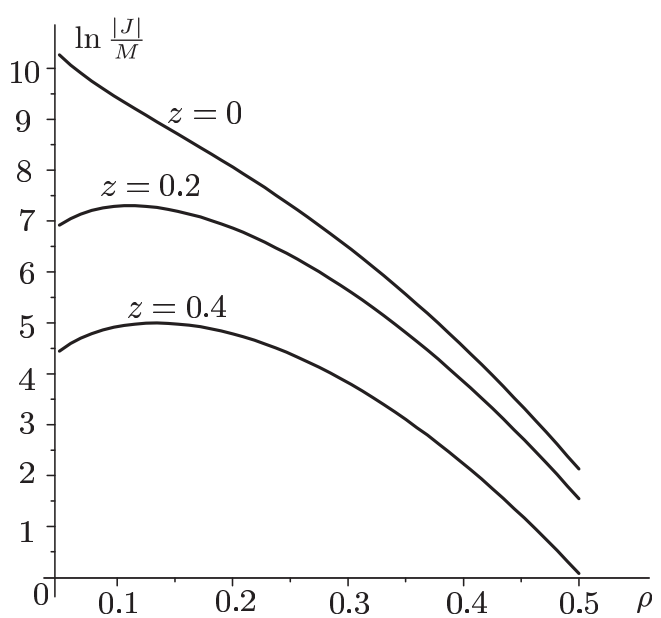

б

Рис. 7. Зависимости величины $\ln (|J| / M)$ от поперечной координаты, рассчитанные по точной формуле (24), в плоскостях $z=$ const в сильном поле $h=100$ для частицы со спином, направленным против поля (а), и скалярной частицы (б). 


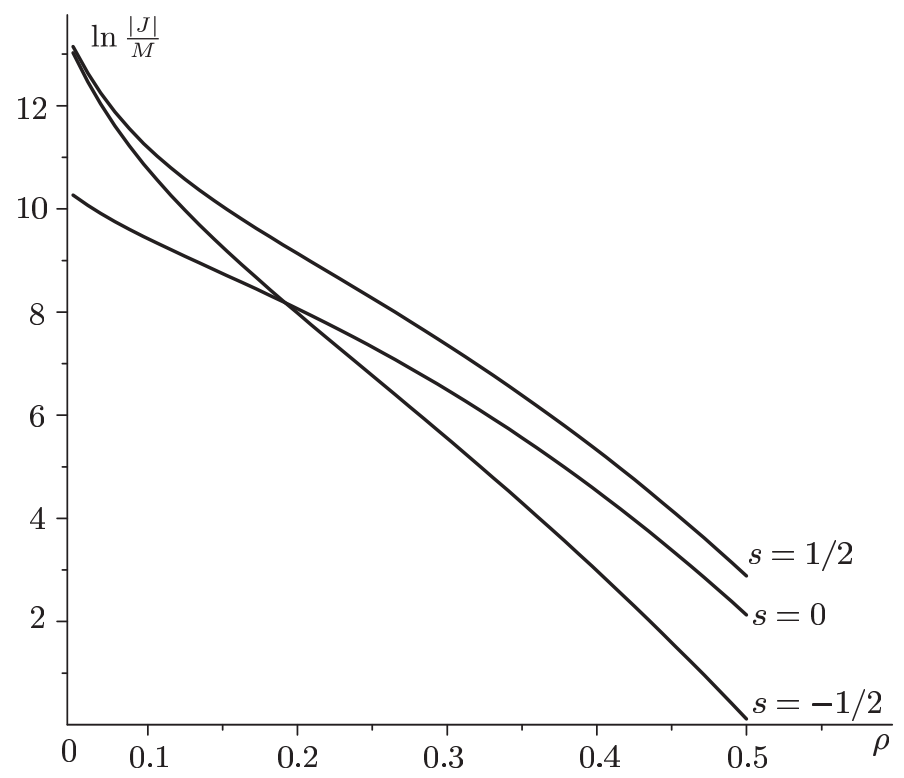

Рис. 8. Зависимость $\ln (|J| / M)$ от поперечной координаты по точной формуле (24) в плоскости $z=0$ для сильного поля $h=100$ при различной ориентации спина (вдоль поля, против поля и в случае спина, равного нулю).

ми они становятся лишь на расстояниях от центра $\tilde{r} \sim 1 / h$, где сам ток в слабом поле $(h \ll 1)$ экспоненциально подавлен. Этот вывод подтверждается при сравнении рис. 6а с рис. 6б, где та же величина $\ln (|J| / M)$ построена для скалярной частицы. При этом сама пространственная часть волновой функции в слабом поле (см. формулу (14)) от спина не зависит. Полученный результат вполне ожидаем, если вспомнить, что в слабом поле $\delta$-яма может связать заряженную частицу только в S-состоянии.

Зависимости величины $\ln (|J| / M)$ от поперечной координаты в плоскостях с различными $z$ в сильном магнитном поле $h=100$, дающие представление о пространственном распределении тока вероятности, приведены на рис. 7а для электрона со спином против поля и на рис. $7 б$ для скалярной частицы. Для расчета здесь была использована точная формула (24).

На рис. 8 в сильном поле в плоскости $z=0$ величина $\ln (|J| / M)$ для скалярной частицы сопоставлена с аналогичными значениями для электрона при различной ориентации его спина. В частности, из рис. 8 видно, что орбитальный и спиновый токи в сильном поле становятся соизмеримыми уже вблизи $\delta$-центра. Как и следовало ожидать, в случае электрона со спином, ориентированным против магнитного поля, ток убывает быстрее по мере удаления от центра, т.е. это движение частицы более локализовано. По-видимому, наиболее интересной для исследования спиновых эффектов является именно область, где орбитальный и спиновый токи оказываются соизмеримыми. 


\section{4. ЗАКЛЮЧЕНИЕ}

Подведем некоторые итоги рассмотрения проблемы учета спина частицы в нерелятивистской задаче. В интенсивном магнитном поле игнорирование спина может привести к некорректным выводам о влиянии поля на целый ряд пороговых процессов [3], [4]. В рамках рассматриваемой темы отметим, что электрический ток, прямо пропорциональный току вероятности, является, в принципе, экспериментально наблюдаемой величиной. Кроме того, он оказывает непосредственное влияние на процессы рассеяния [14]. Из формулы (25) видно, что даже в слабом поле характер особенности тока в окрестности притягивающего $\delta$-центра для частицы со спином имеет характер $\tilde{r}^{-3}$, тогда как для скалярной частицы зависимость оказывается более слабой, вида $\tilde{r}^{-1}$. Для низкоэнергетического рассеяния резерфордовского типа это может иметь принципиальное значение. Отметим также, что орбитальный ток в аналогичной задаче рассчитывался в работе [14] с помощью метода калибровочных преобразований. Это позволило привести волновую функцию к весьма удобному для дальнейших расчетов виду - она становится чисто действительной, и весь орбитальный ток сводится лишь ко второму слагаемому в (21). Однако, поскольку расчет спинового тока в работе [14] не проводился и в ней был также использован приближенный вид волновых функций (см. замечание к формуле (16)), полное сравнение полученных результатов не представляется возможным.

Благодарности. Работа выполнена при частичном участии Программы поддержки ведущих научных школ (грант НШ-65255.2010.2) и РФФИ (грант № 09-0200725 _а). Работа Г.А. Кравцовой частично поддержана Программой поддержки ведущих научных школ (грант НШ-8265.2010.1).

\section{Список литературы}

[1] В. С. Попов, УФН, 174:9 (2004), 921-951.

[2] N. L. Manakov, M. V. Frolov, A. F. Starace, I. I. Fabrikant, J. Phys. B, 33:15 (2000), R141-R214.

[3] В. Н. Родионов, Г. А. Кравцова, А. М. Мандель, Писъма в ЖЭТФ, 75:8 (2002), 435-439.

[4] В. Н. Родионов, Г. А. Кравцова, А. М. Мандель, Докл. АН СССР, 386:6 (2002), 753-755.

[5] Ю.Н. Демков, Г.Ф. Друкарев, ЖЭТФ, 49 (1966), 691-698.

[6] V. N. Rodionov, Phys. Rev. A, 75:6 (2007), 062111; arXiv: hep-ph/0702228.

[7] Р. Фейнман, А. Хибс, Квантовая механика и интеграль по траекториям, Мир, М., 1968.

[8] В. С. Попов, Б. М. Карнаков, В. Д. Мур, ЖКЭТФ, 113:5 (1998), 1579-1605.

[9] В. Н. Родионов, А. М. Мандель, Вестн. МГУ. Сер. Физ. Астрономия, 3 (2001), 25-29.

[10] В. Н. Родионов, Г. А. Кравцова, А. М. Мандель, ТМФ, 145:2 (2005), 198-211.

[11] И. М. Тернов, В.Р. Халилов, В. Н. Родионов, Взаимодействие зарлженных частич с сильным электромагнитным полем, МГУ, М., 1982.

[12] М.В. Федорюк, Асимптотика: интеграль и ряды, Наука, М., 1987.

[13] А.Б. Мигдал, Качественные методы в квантовой теории, Наука, М., 1975.

[14] F. Kh. Chibirova, V. R. Khalilov, Modern Phys. Lett. A, 20:9 (2005), 663-672.

[15] Л. Д. Ландау, Е. М. Лифшиц, Теоретическая физика, т. III, Квантовая механика. Нерелятивистская теория, Наука, М., 1974. 